\title{
Department of Energy \\ Office of Energy Efficiency and Renewable Energy \\ Solid State Lighting Core Technologies
}

\author{
Final Report \\ (April 15, 2011) \\ Award No. DE-EE0000990 \\ Principal Investigator \\ Jiangeng Xue \\ Associate Professor \\ University of Florida \\ Department of Materials Science and Engineering \\ P. O. Box 116400 \\ Gainesville, FL 32611-6400 \\ Phone: (352) 846-3775 \\ Fax: (352) 846-3355 \\ E-mail: jxue@mse.ufl.edu \\ $\underline{\text { Co-Principal Investigators }}$ \\ Elliot Douglas \\ Associate Professor \\ University of Florida \\ Department of Materials Science and Engineering \\ P. O. Box 116400 \\ Gainesville, FL 32611-6400
}




\section{DISCLAIMER}

This report was prepared as an account of work sponsored by an agency of the United States Government. Neither the United States Government nor any agency thereof, nor any of their employees, makes any warranty, express or implied, or assumes any legal liability or responsibility for the accuracy, completeness, or usefulness of any information, apparatus, product, or process disclosed, or represents that its use would not infringe privately owned rights. Reference herein to any specific commercial product, process, or service by trade name, trademark, manufacturer, or otherwise does not necessarily constitute or imply its endorsement, recommendation, or favoring by the United States Government or any agency thereof. The views and opinions of authors expressed herein do not necessarily state or reflect those of the United States Government or any agency thereof. 


\section{Overview}

The overall objective of this project is to demonstrate an ultra-effective light extraction mechanism that can be universally applied to all top-emitting white OLEDs (TE-WOLEDs) and can be integrated with thin film encapsulation techniques. The scope of work proposed in this project includes four major areas: (1) optical modeling; (2) microlens and array fabrication; (3) fabrication, encapsulation, and characterization of TE-WOLEDs; and (4) full device integration and characterization. First, the light extraction efficiency in a top-emitting OLED with or without a microlens array are modeled using wave optics. Second, individual microlenses and microlens arrays are fabricated by inkjet printing of microdroplets of a liquid thiol-ene monomer with high refractive index followed by photopolymerization. Third, high efficiency top-emitting white OLEDs are fabricated, and fully characterized. Finally, optimized microlens arrays are fabricated on TE-WOLEDs with dielectric barrier layers. The overall light extraction efficiency of these devices, as well as its wavelength and angular dependencies, are measured by comparing the efficiencies of devices with and without microlens arrays.

\section{Research Accomplishments}

A. Optical simulation

We have used ray optics to predict the possible light extraction efficiency in top-emitting OLEDs with a hemispherical microlens array. For devices shown in Fig. 1(a), ray optics predicts that the overall light extraction (with the microlens array) can reach $80-90 \%$ with a $\mathrm{Al}$ or $\mathrm{Ag}$ reflecting electrode when the index of refraction for the microlens material matches that of the
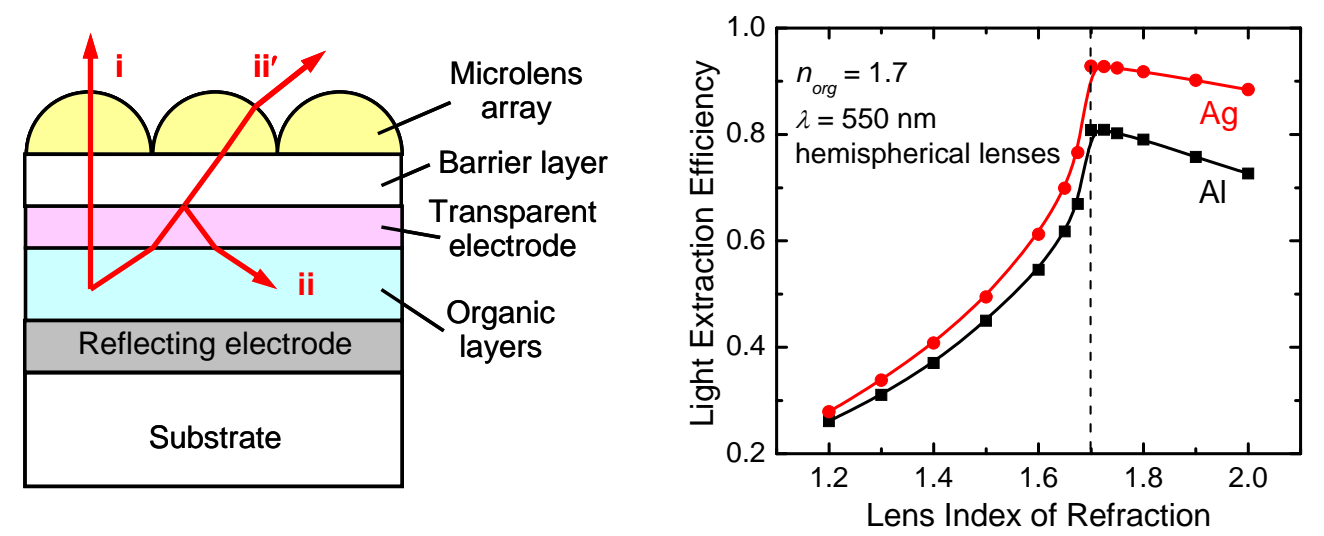

Figure 1. (a) Top-emitting OLED device structure (not to scale). (b) Light extraction efficiency as a function of the microlens refractive index for the top-emitting device with either $\mathrm{Al}$ or $\mathrm{Ag}$ bottom electrode based on ray-optics calculations.

organic layers ( $n_{\text {org }}$ is assumed to be 1.7 in this calculation). As the thickness of the organic layer is typically 100-200 $\mathrm{nm}$ in these OLED devices, less than the visible light wavelength, ray optics is not entirely applicable and we have also used wave optics to more accurately calculate the light extraction efficiency in these devices.

\section{B. Microlens fabrication using an ink-jet printing}

In order to obtain high outcoupling efficiency with microlens arrays, it is necessary for the lenses to have a high contact angle. In order to accomplish an increase in contact angle of the lens material, we have examined various surface treatments for glass substrates. Fluorinated 
alkyl groups are promising candidates because they have a critical surface tension below 20 $\mathrm{mN} / \mathrm{m}$, making them both hydrophobic and oleophobic. Possessing these two aforementioned properties means that hydrocarbons will not spread on surfaces treated with these materials, but rather will bead up.

Two fluorinated and two methylated silane coupling agents were deposited on glass substrates from aqueous alcohol. After this treatment, macrodroplets of a thiol-ene monomer mixture (tri(ethylene glycol) divinyl ether and trimethylolpropane tris(3-mercaptopropionate) in a molar ratio of 3:2) were placed on the treated glass substrate.

\begin{tabular}{|l|l|}
\hline Silane coupling agent & Contact angle \\
\hline (Heptadecafluoro-1, 1, 2, 2, - tetra - hydrodecyl) Trimethoxysilane & $74^{\circ}$ \\
\hline (Heptadecafluoro-1, 1, 2, 2, - tetra - hydrodecyl) Trichlorosilane & $72^{\circ}$ \\
\hline Polydimethylsiloxane, methoxy terminated & $54^{\circ}$ \\
\hline 10-(carbomethoxy) Decyl - Dimethylchlorosilane & $46^{\circ}$ \\
\hline
\end{tabular}

Table 1. Contact angles of lens droplets for different silane coupling agent surface treatments

Fluorinated silane coupling agents show much higher contact angles compared with methylated silane coupling agents (Table 1). However, an OLED cannot be dipped into the aqueous solution as was done for this set of experiments. Hence, in order to apply surface treatment to device, vapor phase deposition of silane coupling agent was employed. By changing deposition time, we were able to control the surface free energy of the glass surface. After vapor phase deposition on the surface of the glass substrate, microlenses were deposited on the substrate via inkjet printing and changes of lens diameter, corresponding to the deposition time, were observed. Utilizing the relationship between dispensing volume and droplet shape, we calculated the diameter of microlens would be around $70 \mu \mathrm{m}$ if the droplet shape is a perfect hemisphere $\left(90^{\circ}\right.$ contact angle) although the diameter of microlens can vary depending on the parameters of inkjet printing system such as nozzle size, driving voltage, and pulse duration. We can reduce lens diameter by increasing deposition time in the closed chamber. Actual contact angle can be measured by taking tilted image of SEM and lens shape can be estimated by measuring lens height as shown in the Fig. 2 (a) and (b).
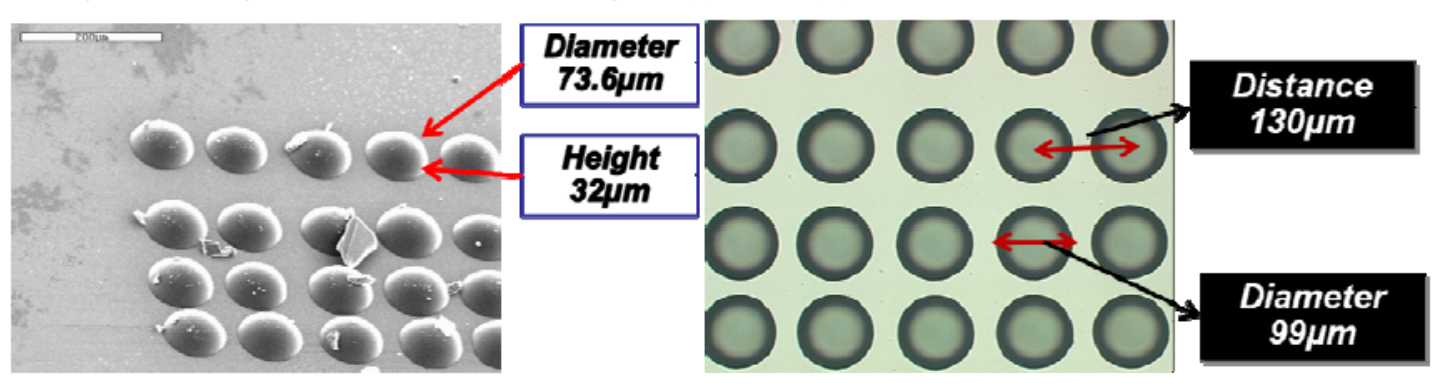

Figure 2. (a) Scanning electron micrograph (angles are tilted) of inkjet-printed microlens arrays. (Heptadecafluoro-1,1,2,2-tetra-hydrodecyl)trimethoxysilane is treated on the glass surface for 3 hours @ $100^{\circ} \mathrm{C}$ before printing. (b) Optical microscopic image of inkjet-printed microlens arrays. (Heptadecafluoro-1,1,2,2-tetra-hydrodecyl)trimethoxysilane is treated on the glass surface for 12 hours @ $100^{\circ} \mathrm{C}\left(40^{\circ} \mathrm{C}\right.$ nozzle temperature is set when inkjet-printing). 
In order to control the refractive index of the lenses, UV curable thiol-alkyne monomer mixtures are employed. Thiols and alkynes are mixed at a 2:1 ratio. By changing sulfur concentration in the networks, we can tailor the index of refraction (Figure 3). For the bottomemission OLEDs, a lens material with a 1.52 index of refraction is needed in order to match the refractive index of the glass substrate. By using decanedithiol (reducing thiol concentration as much as $80 \%$ ) and decadiyne, an index of refraction of 1.52 is obtained after UV curing (wt $\%$ of sulfur is $22 \%$ ). In the case of top-emission OLEDs, lens materials with high refractive index $(\sim 1.7)$ are required. Thiodiethanethiol and heptadiyne monomer mixtures have a sulfur concentration of approximately $48 \%$, resulting in a 1.63 refractive index after polymerization. Work is in progress to increase the refractive index up to 1.7 by adding high refractive index monomers and/or inorganic nanoparticles.

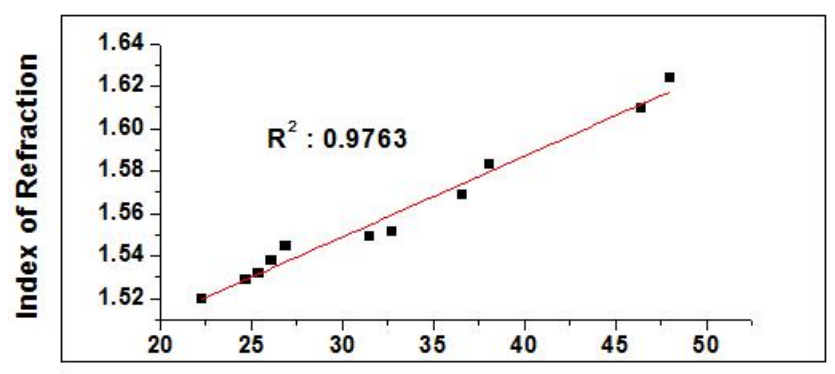

wt\% S

Figure 3. Index of refraction (at $587 \mathrm{~nm}$ ) as a function of wt $\%$ of sulfur for thiol-yne monomers after polymerization

\section{Large area, close-packed hemispherical microlens arrays using a soft-lithography}

We also introduced a soft-lithographic technique to create large area, close-packed hemispherical microlens arrays. Schematic soft-lithographic microlens fabrication process is illustrated in the Fig. 4. Polystyrene (PS) microspheres are used in order to generate a closepacked monolayer on the hydrophilic $\mathrm{Si}$ wafer (coated with $\mathrm{SiO}_{2}$ layer) using a convectivecapillary assembly technique. A polydimethylsiloxane (PDMS) is poured onto the PS monolayer and thermally-cured in the vacuum oven@6 $60^{\circ} \mathrm{C}$ for 2 hours. After removing embedded PS spheres from the cured PDMS, a concave PDMS template could be successfully obtained. In order to create a microlens array on the BOLED, a transparent Norland optical adhesive (NOA)

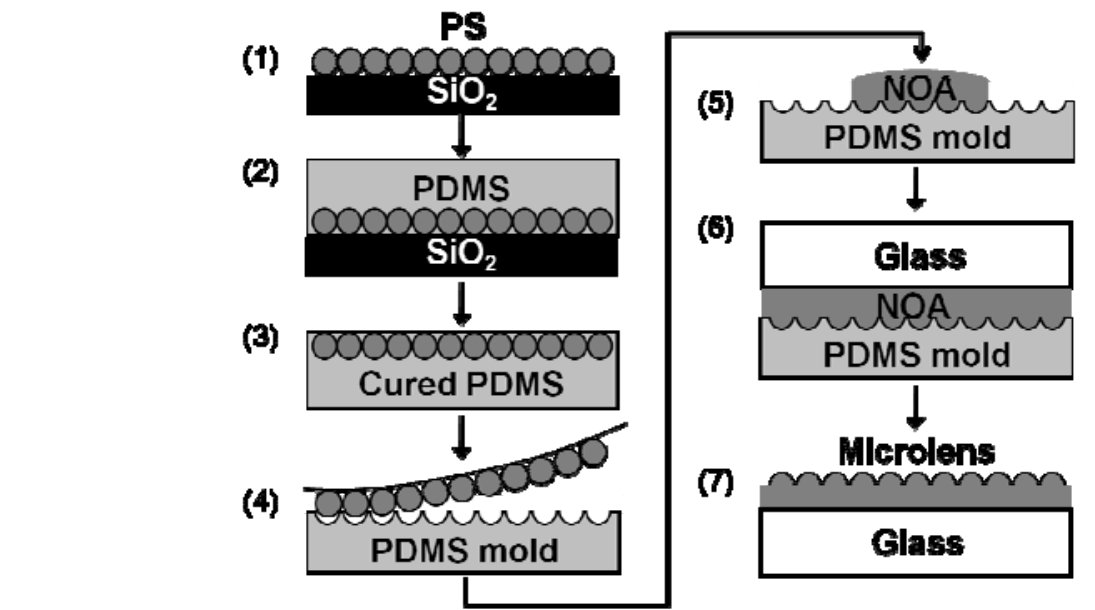

Figure 4. A schematic illustration of soft-lithographic microlens fabrication method. 

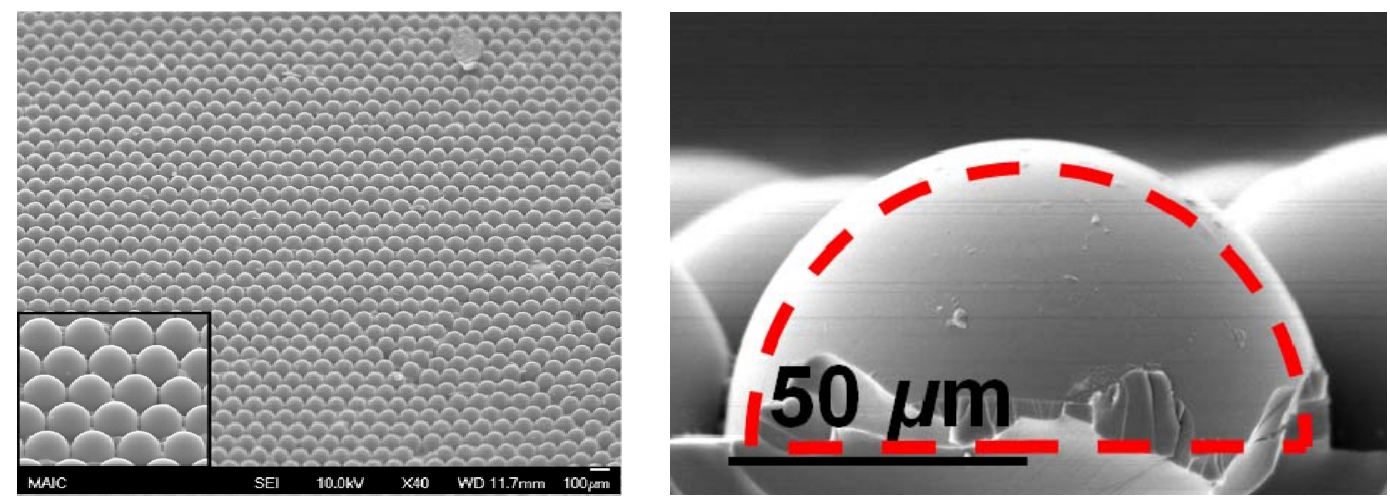

Figure 5. (a) A large area microlens arrays made of $100 \mu \mathrm{m}$ PS spheres. (Inset) A hexagonally closepacked microlens arrays is observed with the measured $F F$ of $(85 \pm 1) \%$. (b) A real hemispherical corss-section image of a microlens $\left(\Phi_{\mathrm{PS}}=100 \mu \mathrm{m}\right)$.

61 is dropped onto the concave surface of PDMS and the glass substrate of a BOLED was placed on top without any pressure. After UV-curing for 3 minutes $\left(365 \mathrm{~nm}, 200 \mathrm{~mW} / \mathrm{cm}^{2}\right)$, the PDMS mold is separated leaving behind a convex microlens array on the glass side of the BOLED.

Figure 5 (a) shows a scanning electron microscopic (SEM) image of a microlens array, which is generated using $100 \mu \mathrm{m}$ size PS spheres. In the inset of Fig. 5 (a), a hexagonally close-

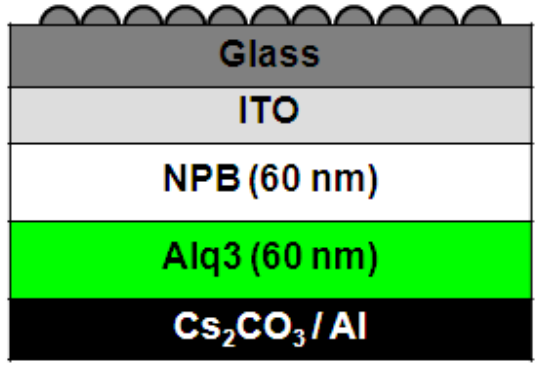

(a)

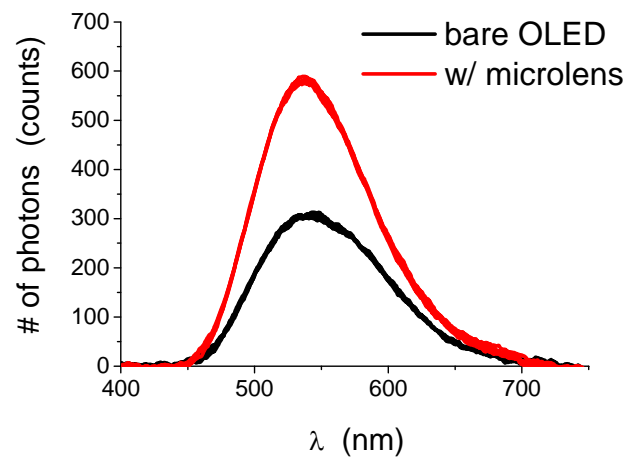

(b)

Figure 6. (a) Schematic device structure of fluorescent bottom-emitting OLEDs (BOLEDs) and microlens arrays attached to the glass surface of OLEDs. (b) Light outcoupling efficiency enhancement measured using an integrating sphere system. The integrated total luminous flux of microlens attached BOLED shows $(70 \pm 7) \%$ higher enhancement than that of bare BOLED.

packed microlens array is clearly shown. The high $F F$ ratio of $(85 \pm 1) \%$ is obtained, which is very close to the ideal $F F$ of two-dimensional hexagonally close-packed spheres $(91 \%)$. A crosssection SEM image of Fig. 5 (b) confirms the hemispherical lens shape with the contact angles $\left(\theta_{C}\right)$ of $\left(85^{\circ} \pm 5^{\circ}\right)$ using $100 \mu \mathrm{m}$ size PS spheres.

The application of such close-packed and hemispherical microlens arrays to the glass substrates of fluorescent OLED, the device structure is shown in the Fig. 6 (a) and the active light-emitting size is $10 \mathrm{~mm} \times 10 \mathrm{~mm}$, resulted in $(70 \pm 7) \%$ enhancement in the light outcoupling efficiency as shown in the Fig. 6 (b).

We also applied microlens to the phosphorescent OLED. In the case of phosphorescent OLED, multiple layer structure is necessary for better exciton/charge confinement, leading to strong optical mode confinement in the organic layers. As a result, the outcoupling efficiency 
enhancement is reduced from $(70 \pm 7) \%$ for the fluorescence OLEDs to $(56 \pm 6) \%$ for phosphorescent OLEDs. Figure 7 (a) shows our bottom-emitting white PHOLED (WOLED) structure and the peak external quantum and power efficiencies of $\eta_{E Q E}=18 \%$ and $\eta_{P}=40 \mathrm{~lm} / \mathrm{W}$ are achieved as shown in the Fig. 7 (b), respectively. We can expect 1.56 times higher outcoupling efficiencies for phosphorescent WOLEDs combined with microlens arrays, presumably achieving peak $\eta_{E Q E}=28 \%$ and $\eta_{P}=62 \mathrm{~lm} / \mathrm{W}$, respectively, as shown in the Fig. $7(b)$.
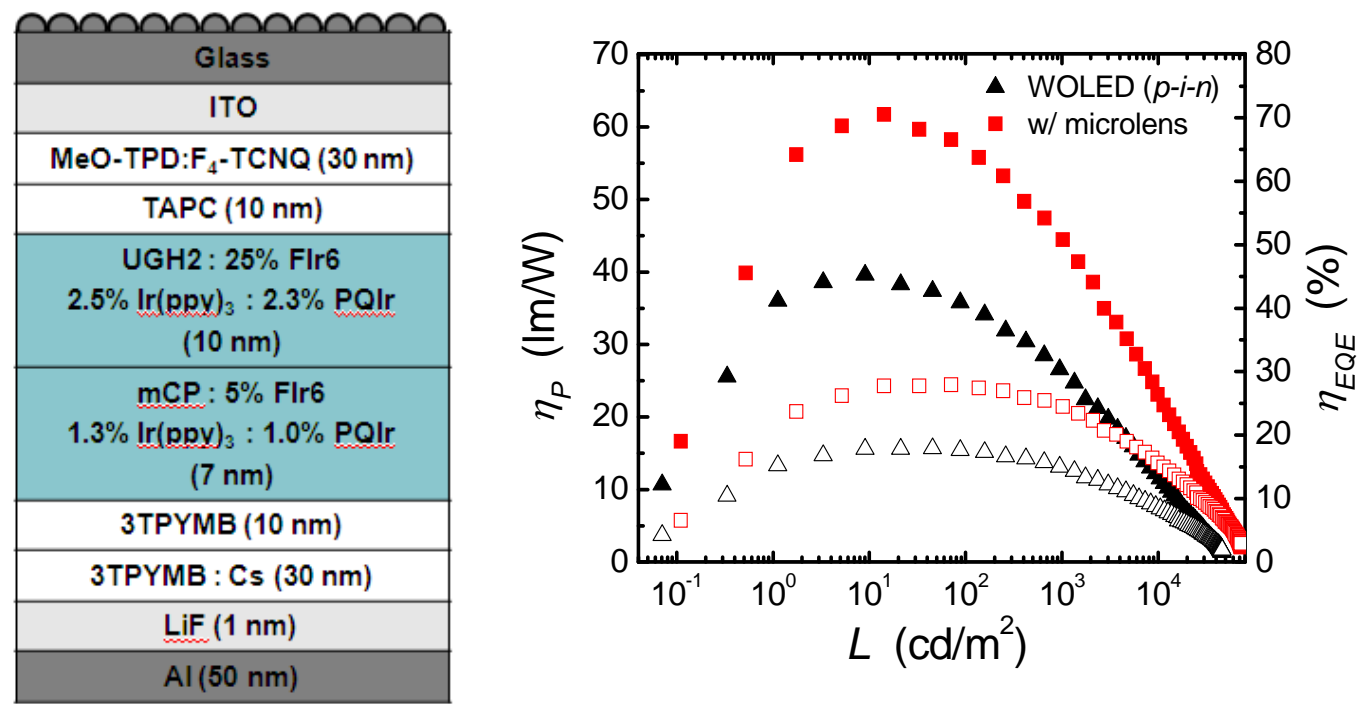

Figure 7. (a) Schematic device structure of bottom-emitting phosphorescent white OLED (WOLED). Microlens arrays can be attached to the glass substrate of the WOLED in order to enhance the outcoupling efficiency (b) external quantum ( $\eta_{E Q E}$, open symbols) and power ( $\eta_{P}$, filled symbols) efficiencies versus luminance $(L)$ for WOLEDs with and without microlens.

\section{Transparent conducting trilayer electrode for a top-emitting OLED}

In order to create a top-emitting organic light-emitting diode (OLED), a transparent electrode is necessary. We have devised an electrode consisting of three layers, molybdenum oxide $\left(\mathrm{MoO}_{3}\right)$, gold $(\mathrm{Au})$, and molybdenum oxide to act as the anode for such a device. We measured both the transmittance and sheet resistance of thin films based on this structure. The films were deposited on glass substrates with the bottom layer of $\mathrm{MoO}_{3}$ fixed at $2.5 \mathrm{~nm}$. The $\mathrm{Au}$ thickness was then varied between $5 \mathrm{~nm}$ and $20 \mathrm{~nm}$ in $5 \mathrm{~nm}$ increments and the top layer of $\mathrm{MoO}_{3}$ was held at $40 \mathrm{~nm}$. Additional films were made holding the bottom layer of $\mathrm{MoO}_{3}$ at 2.5 $\mathrm{nm}$ and $\mathrm{Au}$ at $10 \mathrm{~nm}$, while varying the thickness of the top layer of $\mathrm{MoO}_{3}$ between $20 \mathrm{~nm}$ and $50 \mathrm{~nm}$ in increments of $10 \mathrm{~nm}$. Figure 8 (a) and (b) show the transmittance data for these films compared with both indium tin oxide (ITO) transmittance and the emission spectra of our previous bottom emitting white light indium OLED. While the transmittance does not change significantly with top layer $\mathrm{MoO}_{3}$ thickness, the transmittance does decrease significantly with increasing $\mathrm{Au}$ thickness. While these films do not show as high transmittance as (ITO), transmittance of nearly $90 \%$ can be seen around $600 \mathrm{~nm}$ and only as low as $60 \%$ around $400 \mathrm{~nm}$. 

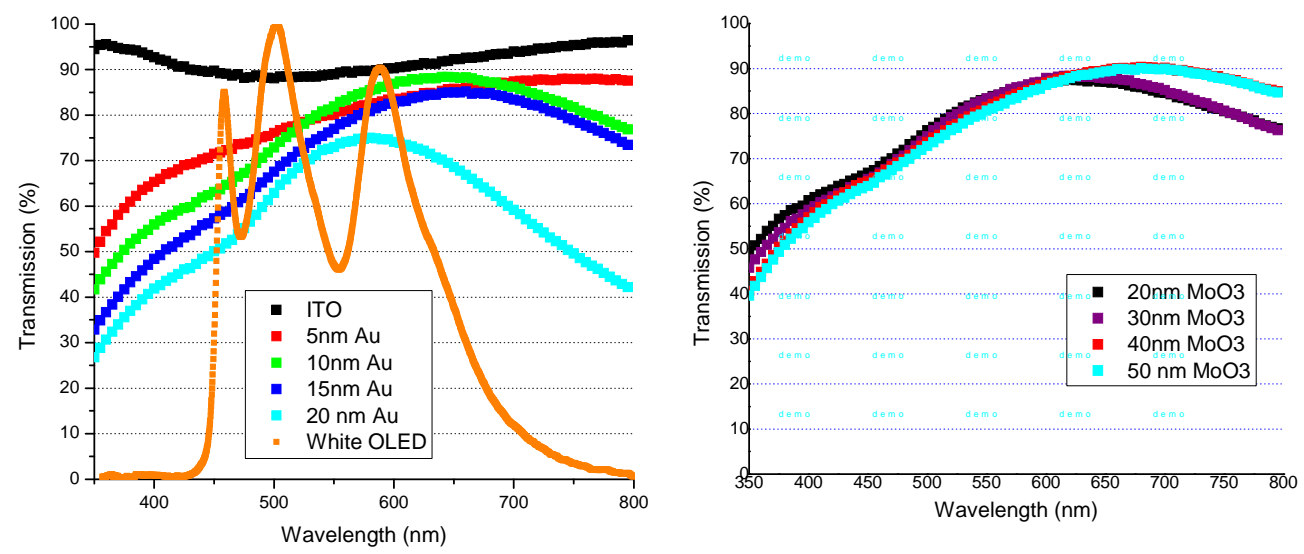

Figure 8. (a) Relative transmittance data of three layer films with varied Au thickness as well as ITO thickness and emission spectra for a white OLED. (b) The relative transmittance of the three layer films by varying the top layer $\mathrm{MoO}_{3}$.

The sheet resistances of the four films with varied Au thickness were then measured using a 4-point probe system. With increasing Au thickness, the sheet resistance of the trilayer continues to decrease. While the film with $5 \mathrm{~nm}$ thick Au shows higher sheet resistance than ITO, once the thickness of this layer is increased to $10 \mathrm{~nm}$ and beyond, the sheet resistance is significantly lower than the ITO. Based on this data, a triple layer consisting of $2.5 \mathrm{~nm} \mathrm{MoO}$, $10 \mathrm{~nm} \mathrm{Au}$, and $40 \mathrm{~nm} \mathrm{MoO}$ will be applied as an anode to a phosphorescent green light emitting devices. The use of $p$ - and $n$ - type current injection layers (MeO-TPD: $\mathrm{F}_{4}-\mathrm{TCNQ}$ and BPhen:Cs, respectively) was necessary to reduce injection barriers into the device. By examining the electroluminescent emission spectrum compared to a device of similar structure, replacing the transparent electrode with ITO and allowing emission through the glass substrate, it can be seen that the new electrode does not cause a significant shift in the emission pattern. The external quantum efficiencies of these two devices shown in the Fig. 9(b) were also compared and the results appear to be similar as well (approximately 12\% EQE for both). These results suggest that this new electrode is a promising candidate for high efficiency top emitting devices.
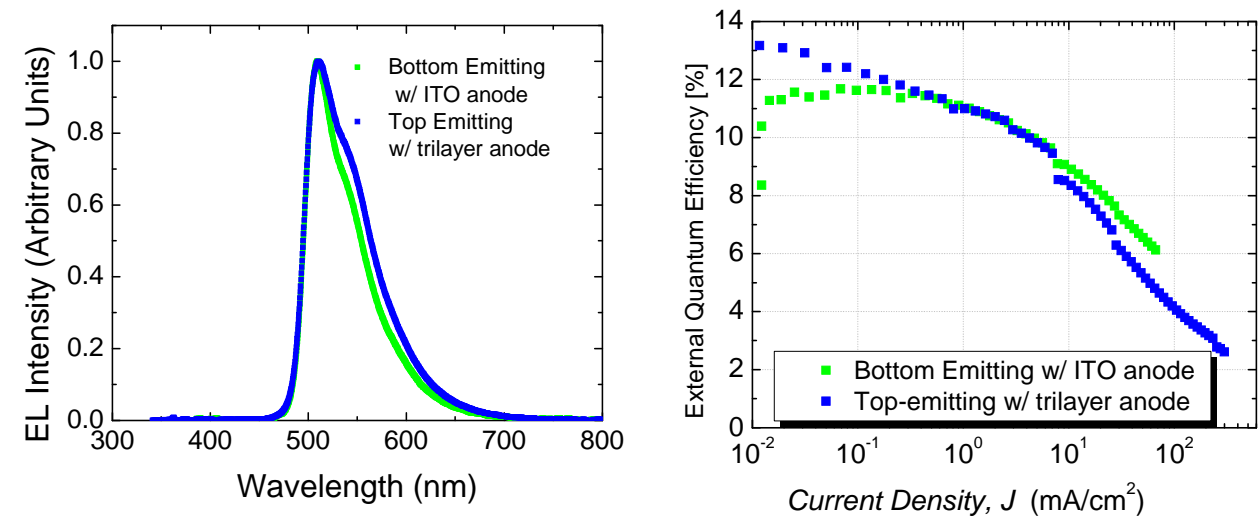

Figure 9. (a) Electroluminescent spectra for the top trilayer electrode and bottom ITO electrode devices, and (b) External quantum efficiencies for the respective devices. 


\section{E. Top-emitting white OLED and integration with microlens arrays}

We applied the oxide/metal/oxide top-emitting electrode to the white OLED structure we have developed earlier with triple phosphorescent dopants. Figure 10 shows the comparison of the efficacy as a function of luminance for several bottom or top-emitting devices. For the top-

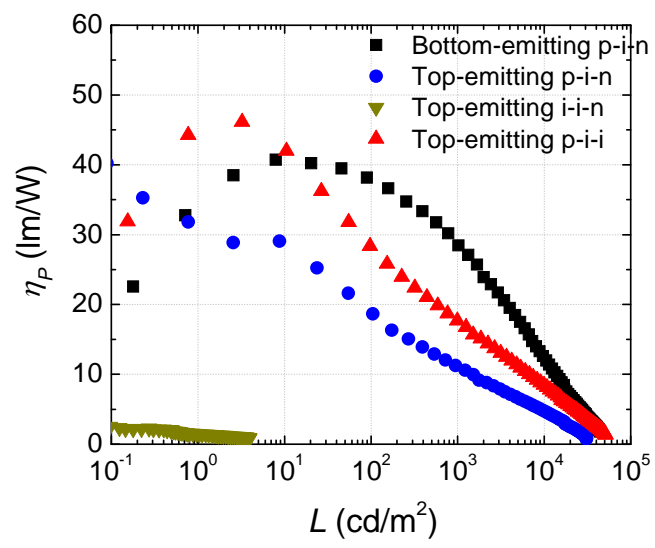

Figure 10. Comparison of bottomand top-emitting white OLEDs with different $\mathrm{p}$ - and $\mathrm{n}$-doping schemes in the charge transport layers.

emitting devices, we have also removed the p- or n-dopants in the conductivity-doped charge injection layers. The top-emitting p-i-i device (which means we did not n-dope the electron transport layer) has a highest efficacy of over $40 \mathrm{~lm} / \mathrm{W}$ at low luminances $\left(\mathrm{L}<10 \mathrm{~cd} / \mathrm{m}^{2}\right)$; however it decreases significantly with the luminance, and $\mathrm{L}=100-1000 \mathrm{~cd} / \mathrm{m}^{2}$, the top-emitting device has $20-40 \%$ lower efficiency than the optimized bottom-emitting device. This suggests that we still need to work out charge balance issue in the bottom-emitting devices. Nevertheless, the p-i-i device is sufficient for the demonstration purpose of the light extraction enhancement with the microlens array.

Large area $\left(1 \mathrm{~cm}^{2}\right)$ top-emitting OLEDs were fabricated, on top of which microlens arrays were applied. During measurement of these devices, it became apparent that the amount of UV exposure the devices received in curing the lenses directly affected the light output of these devices. In order to overcome some of the instability, a new device structure with more chemically stable components was fabricated. This new structure utilizes the same 3TPYMB electron transport, TAPC hole transport, and MeO-TPD: F4-TCNQ hole injection layers of the previous device, but possesses only a single emitting layer consisting of mCP doped with FIr6 (blue), $\operatorname{Ir}(\mathrm{ppy})_{3}$ (green), and PQIr (red). First high efficiency blue green only large area devices were measured with and without microlenses using a stamping method as well as a printing method. Using a luminance meter for comparisons, the enhancement measured in these devices amounted to nearly $140 \pm 10 \%$ for a molded array (see Fig. 11a). Similar enhancement factor was also observed when only the green dopant is present (Fig. 11b). 

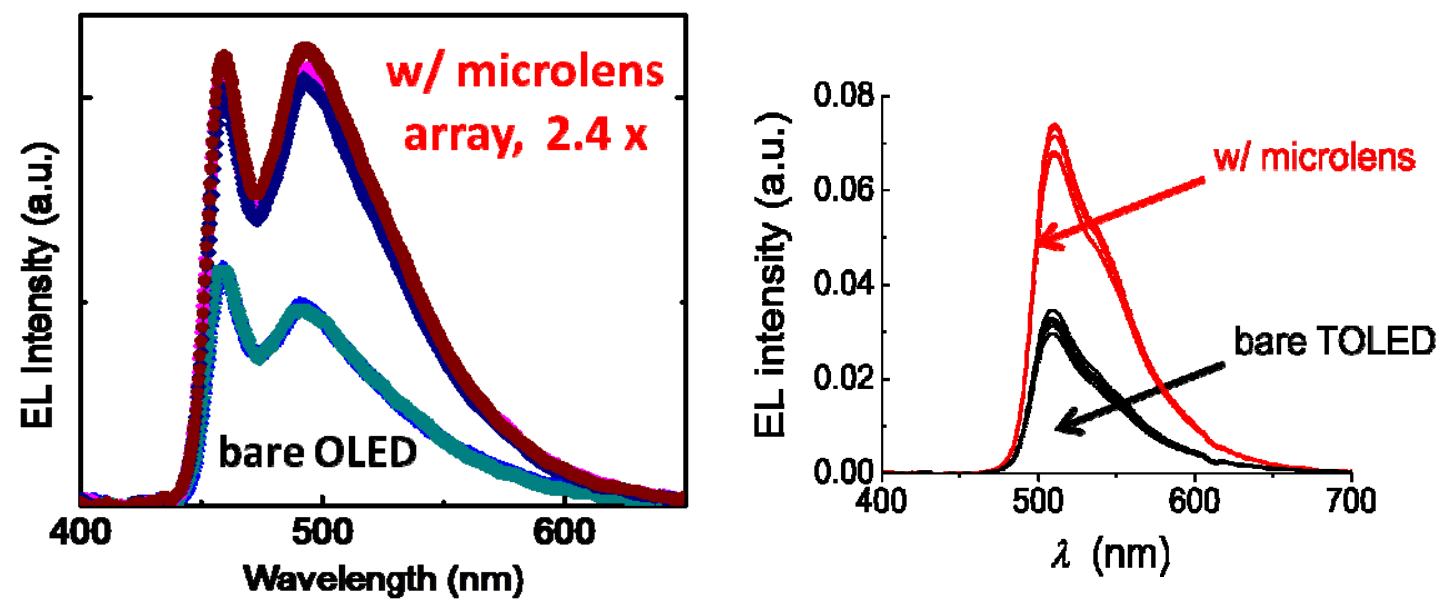

Figure 11 Emission spectra of (a) blue-green and (b) green phosphorescent top-emitting OLEDs with and without a molded microlens, showing a 2.4 times enhancement in the emission intensity

Using the printed microlens array, we were initially not able to obtain consistent results. We attribute that to the possible degradation of the top-emitting OLEDs when the device was exposed to the solvent vapor during silane treatment, as well as degradation during the UV curing of the microlens material. In future studies, we will employ a alumina layer on top of the TE OELD to serve as encapsulation, which would possible solve such degradation issues. In the meantime, we have printed the microlenses on a thin microscope cover glass which was then attached to the top surface of the TE OLEDs using an index matching fluid. In this way, we measured a $90 \%$ enhancement in the light extraction efficiency with such printed microlens array.

Based on these enhancement factors, the power efficiency as a function of luminance for the bare top-emitting white OLED and after applying either a printed microlens array (90\% enhancement) or a molded microlens array (140\% enhancement) is plotted in Fig. 12. The peak power efficiency of the top-emitting white OLED with a microlens array reaches $75-95 \mathrm{~lm} / \mathrm{W}$ (at about $10 \mathrm{~cd} / \mathrm{m}^{2}$ ). At $100 \mathrm{~cd} / \mathrm{m}^{2}$, the power efficiency is $62 \mathrm{~lm} / \mathrm{W}$ (printed lenses) or $80 \mathrm{~lm} / \mathrm{W}$ (moleded lenses). The efficiency rolls off at higher brightnesses, but the device with the printed microlens array still achieves over $40 \mathrm{~lm} / \mathrm{W}$ at $600 \mathrm{~cd} / \mathrm{m}^{2}$ (while the molded lens yields $53 \mathrm{~lm} / \mathrm{W}$ efficacy).

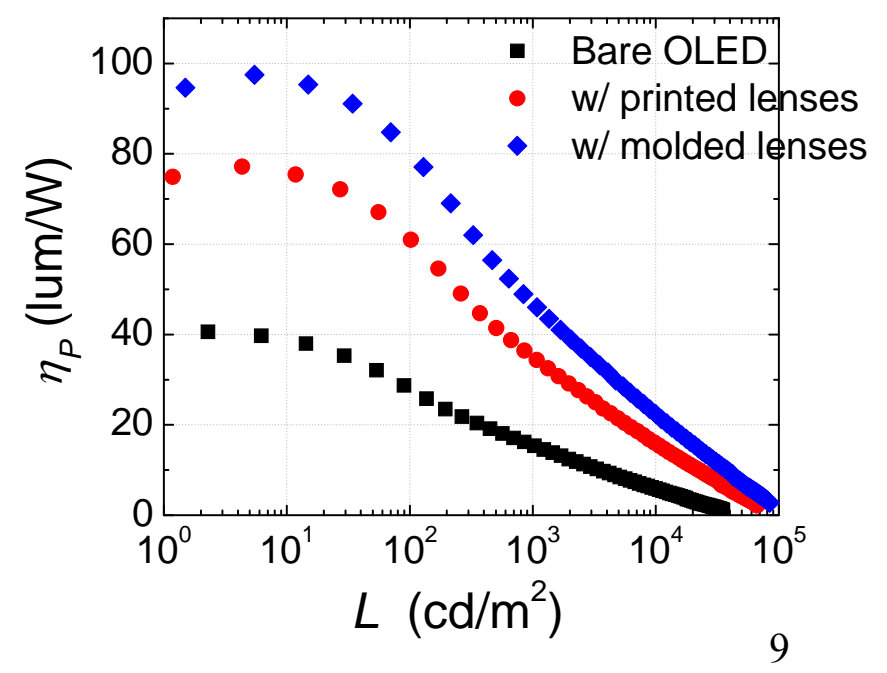

Figure 12. Power efficiency as a function of the luminance for bare top-emitting white OLED and the same device with either a printed or molded microlens array. 


\section{Conclusions}

In conclusion, we have demonstrated the feasibility of applying inkjet printed microlens arrays to enhance the light extraction efficiency of top-emitting white OLEDs. We have shown that the geometry (contact angle) of the printed microlenses can be controlled by controlling the surface chemistry prior to printing the lenses. A $90 \%$ enhancement in the light extraction efficiency has been achieved with printed microlens array on a top-emitting white OLED, which can be further improved to $140 \%$ using a more close-packed microlens array fabricated from a molding process.

Future work will focus on improvement of the microlens fabrication process to improve the array fill factor and the contact angle, as well as use transparent materials with a higher index of refraction. We will also further optimize the procedures for integrating the microlenses on the top-emitting white OLEDs and characterize the overall light extraction enhancement factor when the microlens array is attached. 